\title{
CHARACTERIZATION OF THE CLINIC, IMMUNE CELL RESPONSE AND GENETICS OF PATIENTS WITH CHROMOBLASTOMICOSE IN THE STATE OF MARANHÃO
}

Conceição de M P S Azevedo', Renata R Gomes², Tatiana S Herman², Johnny Ramos do Nascimento', Edith M M Batista', Sirlei Garcia Marques', Matheus C Silva ${ }^{3}$, Daniel Wagner C L Santos ${ }^{4}$ e Vania Aparecida Vicente ${ }^{2}$. 1- UFMA, 2-UFPR, 3-CEUMA, 4-UNIFESP

\section{OBJECTIVE}

To evaluate the cellular immune response in patients with chromoblastomycosis and to analyze the patients with more severe disease and a more reserved prognosis regarding the mutation in the CARD9 gene.

\section{METHODS}

A total of 89 patients were followed at the Reference Center for Infectious Diseases (RECIPD), with diagnosis of chromoblastomycosis by direct examination, culture and histopathology. IL-2, IL-4, IL-6, IL-10, IFN- $\gamma$, TNF- $\alpha$ and IL-17A cytokines were quantified by Cytometric Bead Array (CBA) Mouse Th1 / Th2 / Th17 cytokines in 89 patients. From the blood samples from 28 patients, the extraction of genomic DNA was performed using the Purelink Genomic DNA kit (Invitrogen). After DNA extraction and primer construction, standardization of the polymerase chain reaction (PCR) was performed to amplify the coding region CARD9. The sequencing reaction was performed using BigDye Terminator Cycle Kit v.3.1 (Applied Biosystems, Foster City, CA, USA). The editing and analysis of the sequences obtained was performed with the aid of BioEdit (HALL, 1999) and MEGA version 7 (Kumar, Stecher, and Tamura 2015), which were compared with the wild-type CARD9 gene sequence (Ensemb number: ENSG00000187796 ) and sequences from the GenBank database (NCBI -http: //www.ncbi.nlm.nih.gov/), with the aid of the MAFFT program (MISAWA; MIYATA, 2002), alignment and visual inspection with aid of the MEGA program version 7. The Q295X mutation in the CARD9 gene, characterized by the exchange of $C$ by $T$ in codon 295 of exon 6 , was evaluated.
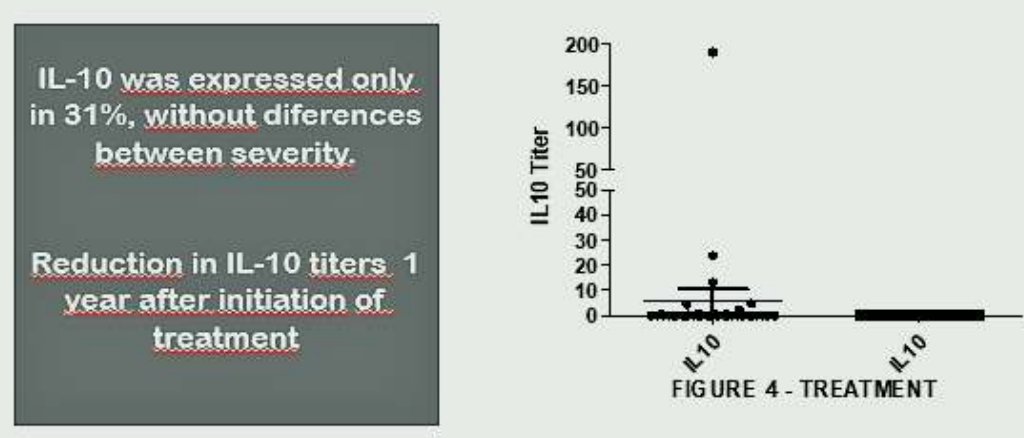

\section{RESULTS}

It was observed that the majority of patients were male (79 patients $(88.76 \%)$ ); with a mean age at diagnosis of 58.1 years (ranging from 33 to 87 years) and standard deviation of 11.49. Mutation in Exon 6 of the CARD9 gene was not observed in the patients.
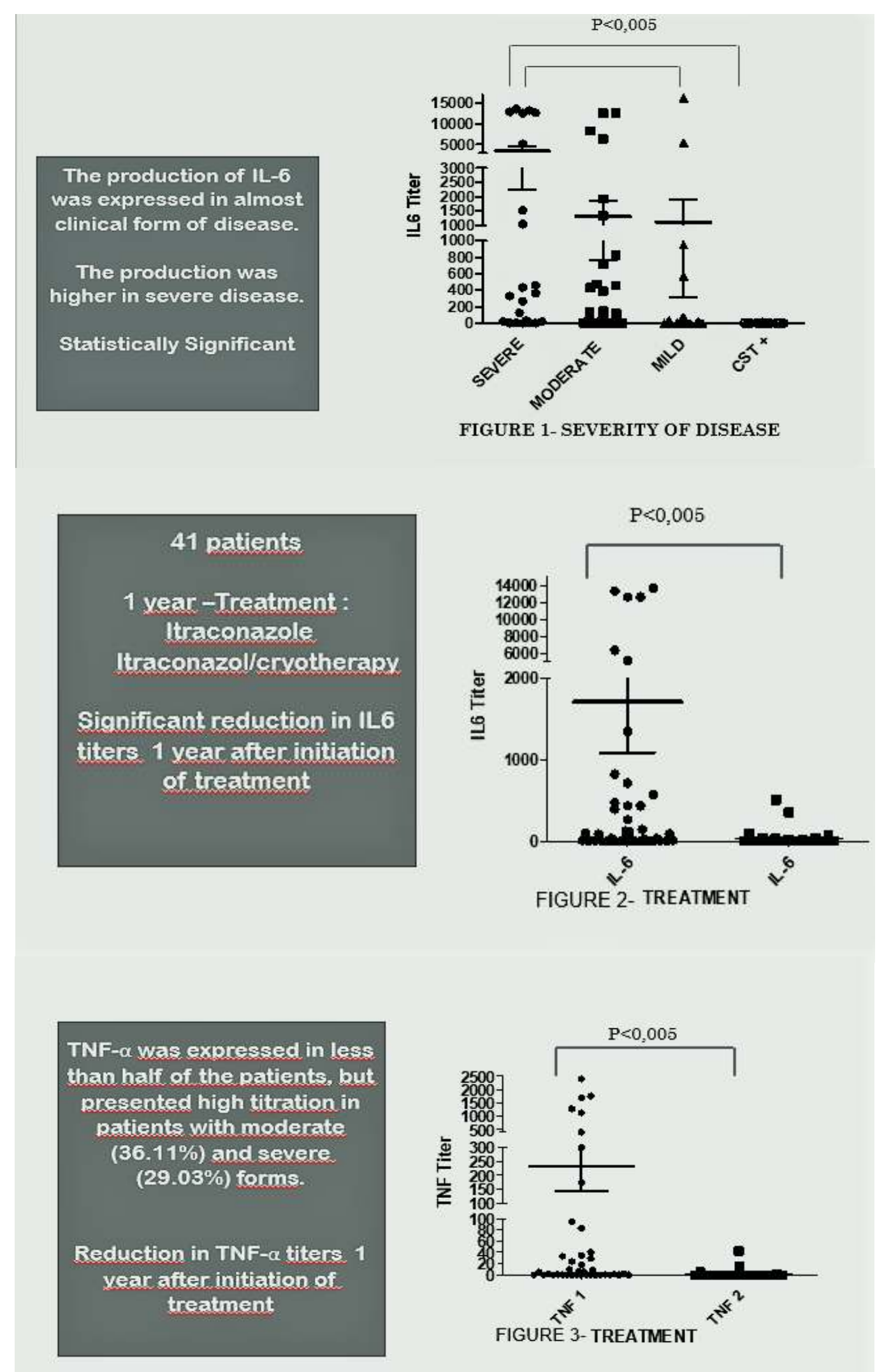

\section{CONCLUSION}

The fact that IL- 6 and TNF- $\alpha$, acute phase markers, are present in high titers in patients with moderate and severe forms, infers an intense pro-inflammatory activity in all stages of the disease, denoting its recurrent character. The association of the Th1 pattern with milder forms of the disease and the Th2 pattern with severe forms was observed again, whereas the Th17 response, characteristic of patients with chronic infections, was not evidenced in this series. Mutation in CARD9 was not observed, indicating that other mechanisms may be involved in the abstention of Th17. 